\title{
Effects of in ovo feeding of creatine pyruvate on the hatchability, growth performance and energy status in embryos and broiler chickens
}

\author{
M. M. Zhao, T. Gao, L. Zhang, J. L. Li, P. A. Lv, L. L. Yu, F. Gao ${ }^{\dagger}$ and G. H. Zhou \\ College of Animal Science and Technology, Jiangsu Key Laboratory of Animal Origin Food Production and Safety Guarantee; Jiangsu Collaborative Innovation Center \\ of Meat Production and Processing, Quality and Safety Control, Nanjing Agricultural University, Nanjing 210095, China
}

(Received 9 May 2016; Accepted 16 January 2017; First published online 21 February 2017)

\begin{abstract}
The effects of in ovo feeding (IOF) of creatine pyruvate (CrPyr) on the hatchability, growth performance and energy status of embryos and broilers (Arbor Acres) were investigated. Five treatments were arranged as non-injected treatment (Control), $0.6 \mathrm{ml}$ physiological saline (0.75\%) injected treatment (Saline), and IOF treatments injected with $0.6 \mathrm{ml}$ physiological saline (0.75\%) containing 3, 6 or $12 \mathrm{mg} \mathrm{CrPyr}\left(\mathrm{CrPyr}_{3} \mathrm{CrPyr}_{6}\right.$ or $\left.\mathrm{CrPyr}_{12}\right)$ into the amnion per fertile egg on day 17.5 of incubation. After hatching, 80 male chicks from each treatment with similar weight close to the average BW of their pooled group were selected and randomly assigned into eight replicates of 10 chicks each. The results showed that the hatchability was not affected among groups, whereas the hatching weight of broilers in $\mathrm{CrPyr}_{12}$ was significantly higher than the control and saline groups $(\mathrm{P}<0.05)$. At 21 day post-hatch, the BWs of broilers in $\mathrm{CrPyr}_{6}$ and $\mathrm{CrPyr}_{12}$ were increased relative to the control and saline groups $(\mathrm{P}<0.05)$. Chickens in $\mathrm{CrPyr}_{6}$ and $\mathrm{CrPyr}_{12}$ exhibited higher BW gain and feed intake than the control and saline groups during 8 to 21 days post-hatch and the entire experiment period $(\mathrm{P}<0.05)$. Compared with the control and saline groups, the total and relative weight of pectoral muscle of embryos or chickens were greater in $\mathrm{CrPyr}_{6}$ and $\mathrm{CrPyr}_{12}$ at $19^{\text {th }}$ day of incubation $(19 \mathrm{E})$, hatch, 3 and 21 days post-hatch $(\mathrm{P}<0.05)$. The concentrations of glucose and glycogen in liver were increased in $\mathrm{CrPyr}_{6}$ and $\mathrm{CrPyr}_{12}$ at $19 \mathrm{E}$ and hatch $(\mathrm{P}<0.05)$. Neither glycogen nor glucose concentration in pectoral muscle was altered among treatments $(\mathrm{P}>0.05)$. Irrespective of dosage, the concentrations of creatine and phosphocreatine, and activities of creatine kinase in embryos were enhanced in CrPyr treatments at $19 \mathrm{E}$ when compared with the control and saline groups $(\mathrm{P}<0.05)$. The activities of glucose-6-phosphatase in liver in $\mathrm{CrPyr}_{6}$ and $\mathrm{CrPyr}_{12}$ treatments were higher than the control and saline groups at $19 E(\mathrm{P}<0.05)$. In conclusion, these results indicated that IOF of CrPyr, especially at the level of $12 \mathrm{mg} / \mathrm{egg}$, could improve energy status of embryos and hatchlings, which was useful for enhancing hatching weight, BW and pectoral muscle weight until the end of the experiments at 21 days post-hatch in broilers.
\end{abstract}

Keywords: in ovo feeding, creatine pyruvate, growth performance, energy status, broilers

\section{Implications}

Nowadays, early nutritional regulation (in ovo feeding (IOF) of exogenous nutrients) has been indicated to offer the promise of sustaining progress in production efficiency of commercial poultry. The present study showed that IOF of creatine pyruvate (CrPyr) (which contains pyruvic acid molecularly bound to creatine $(\mathrm{Cr})$ at a concentration ratio of $40: 60$ ), especially at the level of $12 \mathrm{mg} / \mathrm{egg}$, could improve hatching weight, BW and pectoral muscle weight until 21 days post-hatch in broilers. These findings provide a basis for future work on the use of CrPyr to solve the deficiency of energy reserves during the late embryogenesis.

\footnotetext{
${ }^{\dagger}$ Email: gaofeng0629@sina.com
}

\section{Introduction}

Unlike mammals, avian species, which do not have a continuous maternal energy supply, possess limited nutrient and energy deposits in the fertile egg to support embryonic and neonatal growth. During pre-hatch period, glucose and glycogen are preferentially utilized as the main energy sources for the nutrition of avian embryos (Shafey et al., 2012). However, the glycogen reserves are significantly depleted in order to meet the high energy demand toward the end of incubation. This may consequently force the embryo to mobilize more muscle protein for gluconeogenesis thereby inhibiting early growth and development (Chen et al., 2009; Noy and Uni, 2010). In addition, under commercial industry practices, chicks are deprived of feed and 
water for 24 to $72 \mathrm{~h}$ because of variation in hatch time, chick handling, and transportation time (Willemsen et al., 2010). Delaying access to feed and water for 36 to $72 \mathrm{~h}$ aggravates the deficiency of energy and leads to irreversible damage to broilers, such as retard of BW, depression of intestinal development and lower pectoral muscle weight (Kornasio et al., 2011; Lamot et al., 2014). Therefore, the few days pre- and post-hatch are crucial for the development of hatchlings, suggesting that the improvement of energy storage during this period may promote subsequent growth performance of chickens.

In ovo feeding is a technique of administrating exogenous nutrients into the amnion of the late-term avian embryos, as the embryos can orally consume the amniotic fluid and then absorb the added nutrients by the intestine before piping (Uni and Ferket, 2004). Several attempts have revealed that IOF of exterior nutrient substances such as carbohydrate, amino acids or protein could increase hatching weight, liver glycogen reserves, marketing weight and breast muscle yield (Uni and Ferket, 2004; Uni et al., 2005; Foye et al., 2006a; Tangara et al., 2010). Therefore, the IOF, an inspiring insight for perinatal nutrition of poultry embryos, may be beneficial to overcome the restriction of finite energy in late-term bird embryos.

Creatine pyruvate is an organic compound, which contains pyruvic acid molecularly bound to $\mathrm{Cr}$ at a concentration ratio of $40: 60$ (Chen et al., 2012). Pyruvate, which works as an intermediate product of carbohydrate, protein and lipid, can modulate energy metabolism through the glycolytic/gluconeogenesis pathway and the Krebs cycle; while $\mathrm{Cr}$, a nitrogen containing compound, can be phosphorylated as phosphocreatine $(\mathrm{PCr})$, which are directly involved in the muscle energy buffering system by transferring a phosphate group to ADP to replenish ATP (Chen et al., 2011; Allen, 2012). Meanwhile, previous study in our lab demonstrated that combined IOF of creatine monohydrate and glucose during the last stage of incubation had synergistic effects on elevating the glycogen reserves in liver and increasing the concentrations of $\mathrm{Cr}$ and $\mathrm{PCr}$ in muscle of embryos and hatchlings (Zhang et al., 2016). Thus, we hypothesize that IOF of CrPyr would enhance energy reserves and support the growth of avian embryos and neonates. Therefore, the objectives of the present study were to evaluate the effects of IOF of CrPyr on hatchability, growth performance and energy status of embryos and broilers from 19 days pre-hatch until 21 days post-hatch.

\section{Material and methods}

\section{Egg incubation}

All procedures were approved by the Institutional Animal Care and Use Committee of Nanjing Agricultural University. A total of 1400 fertile broiler eggs (Arbor Acres) from a laying flock at 34 weeks of age were pre-weighed and selected from a commercial hatchery with an average weight of $62.17 \pm 1.63 \mathrm{~g}$ (range $=60$ to $65 \mathrm{~g}$ ). Eggs were then randomly assigned in a microcomputer automatic incubator (ZCA-A; Zhicheng Incubation Equipment Co., Ltd., Dezhou, China) under routine conditions $\left(37.8 \pm 0.1^{\circ} \mathrm{C}\right.$ of temperature and $60 \%$ of relative humidity) and turned through $270^{\circ}$ every $1.5 \mathrm{~h}$ until $19^{\text {th }}$ day of incubation (19 E). On embryonic day 6 , eggs were candled and unfertilized eggs were removed from the incubator.

\section{In ovo feeding procedure}

All injected solutions were freshly prepared on the day of injection. The CrPyr (Ju sheng Technology Co., Ltd., Wuhan, China) was dissolved in physiological saline $(0.75 \%)$ to achieve concentrations of 5, 10 or $20 \mathrm{mg} \mathrm{CrPyr} / \mathrm{ml}$, respectively. Then solutions were sterilized by filtration through a $0.22 \mu \mathrm{m}$ membrane filter and then subsequently kept in the incubator at $37.8^{\circ} \mathrm{C}$. At the end of embryonic day 16 , all eggs were illuminated again and non-viable eggs were removed. Of the remaining eggs, 1200 available eggs with similar weight within $\pm 1 \%$ of the mean weight $(56.64 \pm 0.51 \mathrm{~g})$ were randomly divided into five treatment groups with eight replicates of 30 eggs each. In total, 40 incubator trays were used, and each tray was taken as one replicate. Treatment 1 was non-injected group (Control), treatment 2 was $0.6 \mathrm{ml}$ physiological saline $(0.75 \%)$ injected group (Saline), treatments 3 to 5 were injected with $0.6 \mathrm{ml}$ physiological saline $(0.75 \%)$ solution containing 3,6 or $12 \mathrm{mg}$ CrPyr/egg $\left(\mathrm{CrPyr}_{3}\right.$, $\mathrm{CrPyr}_{6}$ or $\left.\mathrm{CrPyr}_{12}\right)$, respectively. On embryonic day 17.5 , the operation procedures were performed as described in detail by Uni et al. (2005) and Zhai et al. (2011b). The location of the amnion was identified by candling and the injection place was disinfected with $75 \%$ ethyl alcohol at the surface of the large end of the egg. A hole was then punched using a needle and the IOF solution was injected into the amnion using a 21-gauge needle (the syringe was used in a disposable way) to a depth of about $2.49 \mathrm{~cm}$. Immediately after the injection experiment, the injected holes on the eggs were sealed with petroleum wax, and transferred to hatching trays. All eggs were exposed outside the incubator for $<30 \mathrm{~s}$ to complete the IOF procedure. Until hatch, all eggs were incubated according to the routine procedure. The remaining eggs served as the non-injected control were subjected to the same handling procedures as the IOF groups.

\section{Animal husbandry}

Upon hatch, the number of hatchlings within each treatment was recorded. Hatchability was calculated as $(\%)=$ (number of hatchlings/number of fertile eggs) $\times 100$. All male hatched chicks from one treatment were pooled and weighted. In all, 80 male chicks with similar weight close to the average BW of their pooled group were selected and randomly assigned into eight replicates of 10 chicks within each treatment. In total, 40 pens were provided for the five treatment chicks, with each replicate allocated for a pen $(110 \times 60 \times 50 \mathrm{~cm})$. The chickens were allowed free access to feed and water in three-layer cages in a temperature-controlled room and the temperature was set at $32^{\circ} \mathrm{C}$ to $34^{\circ} \mathrm{C}$ for the first 3 days and then reduced by $2^{\circ} \mathrm{C}$ to $3^{\circ} \mathrm{C}$ per week. All birds were reared 
Table 1 The composition and calculated nutrient levels of the basal diets

\begin{tabular}{lr}
\hline \hline Items & Value \\
\hline Ingredients (\%) & \\
Corn & 57.61 \\
Soybean meal & 31.00 \\
Corn gluten meal & 3.29 \\
Soybean oil & 3.11 \\
Limestone & 1.20 \\
Dicalcium phosphate & 2.00 \\
L-lysine & 0.34 \\
DL-methionine & 0.15 \\
Salt & 0.30 \\
Premix & 1.00 \\
Calculated nutrient levels & \\
ME (MJ/kg) & 12.56 \\
CP (\%) & 21.10 \\
Ca (\%) & 1.00 \\
Available phosphorus (\%) & 0.46 \\
Lysine (\%) & 1.20 \\
Methionine (\%) & 0.50 \\
Methionine + cysteine (\%) & 0.85 \\
\hline \hline
\end{tabular}

$\mathrm{ME}=$ metabolizable energy.

${ }^{1}$ Premix provided per kilogram of diet: retinyl acetate for vitamin A, $12000 \mathrm{IU}$; cholecalciferol for vitamin $D_{3}, 2500 \mathrm{IU}$; $\mathrm{DL}-\alpha$-tocopheryl acetate for vitamin $\mathrm{E}_{4}$ $20 \mathrm{IU}$; menadione sodium bisulfate, $1.3 \mathrm{mg}$; thiamin, $2.2 \mathrm{mg}$; riboflavin, $8.0 \mathrm{mg}$; nicotinamide, $40 \mathrm{mg}$; choline chloride, $400 \mathrm{mg}$; calcium pantothenate, $10 \mathrm{mg}$; pyridoxine $\mathrm{HCl}, 4 \mathrm{mg}$; biotin, $0.04 \mathrm{mg}$; folic acid, $1 \mathrm{mg}$; vitamin $\mathrm{B}_{12}$ (cobalamin), $0.013 \mathrm{mg}$; Fe (from ferrous sulfate), $80 \mathrm{mg}$; Cu (from copper sulfate), $8.0 \mathrm{mg}: \mathrm{Mn}$ (from manganese sulfate), $110 \mathrm{mg}$; $\mathrm{n}$ (from zinc sulfate), $60 \mathrm{mg}$; I (from calcium iodate), $1.1 \mathrm{mg}$; Se (from sodium selenite), $0.3 \mathrm{mg}$.

under incandescent white light with a light schedule of $23 \mathrm{~h}$ light and $1 \mathrm{~h}$ dark according to Zhang et al. (2014). The diets were formulated to meet the nutrient requirements of Arbor Acres broiler chickens (Table 1). At 7 and 21 days, birds were weighed after feed deprivation for $12 \mathrm{~h}$ and feed intake was recorded by replicate to calculate BW gain, feed intake and feed/gain ratio.

\section{Tissue sampling}

The entire embryos were removed and cleaned of yolk sac and membrane after the eggs were opened from air chamber at $19 \mathrm{E}$. They were then euthanized with sodium pentobarbital $(20 \mathrm{mg} / \mathrm{kg}$ of BW; Beijing Chemical Co., Beijing, China). One male embryo was randomly selected by observing the morphology of the gonads (embryo with two tubular shaped gonads of about equal length was identified as male) from each replicate (eight per treatment) according to the method of Burke (1994). Then the yolk-free body and entire pectoral muscle were weighed and recorded. The samples of liver and pectoral muscle were collected and frozen immediately in liquid nitrogen for further analysis.

On the age of hatch, 3, 7 and 21 days post-hatch, one bird (eight birds per treatment) with a BW close to the average BW of the replicate was selected and weighed, then killed by cervical dislocation. The entire pectoral muscle was obtained and weighed. Moreover, samples of liver and pectoral muscle were obtained and frozen in liquid nitrogen until analysis.
Liver and muscle glycogen and glucose analysis

The concentrations of glycogen in liver and pectoral muscle were estimated with a 1200 UV spectrophotometer (Mapada Instruments Co. Ltd., Shanghai, China) according to the directions of commercially available liver glycogen/muscle glycogen detection kits (Nanjing Jiancheng Bioengineering Institute, Nanjing, China). The concentration of glucose was measured using commercial glucose oxidase kits (Shanghai RongSheng Biotech Co. Ltd., Shanghai, China).

\section{Concentrations of creatine and phosphocreatine in pectoral muscle}

The concentrations of $\mathrm{Cr}$ and $\mathrm{PCr}$ in pectoral muscle were determined by reverse-phase-HPLC according to Li et al. (2016). Briefly, each $200 \mathrm{mg}$ frozen muscle sample was homogenized in $2 \mathrm{ml}$ ice-cold $5 \% \mathrm{HClO}_{4}$ for $1 \mathrm{~min}$ and centrifuged at $10000 \times \mathrm{g}$ at $4^{\circ} \mathrm{C}$ for $10 \mathrm{~min}$ after being lixiviated in an ice bath for $15 \mathrm{~min}$. The supernatant was then mixed with $900 \mu \mathrm{l}$ of $0.8 \mathrm{M} \mathrm{K}_{2} \mathrm{CO}_{3}$. The mixture was centrifuged for $10 \mathrm{~min}$ at $10000 \times \mathrm{g}$ at $4^{\circ} \mathrm{C}$ again after being neutralized in an ice bath for $10 \mathrm{~min}$. Next, the supernatant was filtered through a $0.45 \mu \mathrm{m}$ filtration membrane and injected into the Waters-2695 Alliance HPLC system (Waters, Milford, MA, USA) equipped with an integrated auto-sampler. The analytical column used in the experiments was Waters SunFire C18 $(250 \times 4.6 \mathrm{~mm}, 5 \mu \mathrm{m}$; Waters) with a column temperature of $25^{\circ} \mathrm{C}$. The mobile phase consists of $2 \%$ methyl cyanides and $98 \% \mathrm{KH}_{2} \mathrm{PO}_{4}$ buffer $(29.4 \mathrm{mM})$ and the flow rate was kept at $1.0 \mathrm{ml} / \mathrm{min}$. Other chromatographic conditions were set as follows: UV detection wavelength, $210 \mathrm{~nm}$; and the injection volume, $20 \mu \mathrm{l}$. The standard curves were established according to the method reported by Zhang et al. (2010).

Determination of creatine kinase activity in pectoral muscle For each bird, $200 \mathrm{mg}$ frozen muscle sample was weighed and homogenized in a centrifuge tube with $1.8 \mathrm{ml}$ of $0.75 \%$ saline, and then centrifuged at $3500 \times \mathrm{g}$ for $10 \mathrm{~min}$ at $4{ }^{\circ} \mathrm{C}$. The supernatant was used for assaying the activity of creatine kinase with commercial kits (Nanjing Jiancheng Bioengineering Institute, Nanjing, China). The result was normalized against total protein concentration in each sample. The concentrations of protein in tissue extracts were estimated according to the manufacturer's protocol of total protein quantitative assay kits (Nanjing Jiancheng Bioengineering Institute, Nanjing, China).

\section{Determination of glucose-6-phosphatase activity in liver}

The activity of glucose-6-phosphatase in liver was determined using modified procedures described by Donaldson and Christensen (1991). Each $150 \mathrm{mg}$ liver sample was homogenized in a $0.25 \mathrm{M}$ sucrose solution $(1 \mathrm{~g}$ liver $/ 10 \mathrm{ml})$ and centrifuged at $14000 \times \mathbf{g}$ at $4^{\circ} \mathrm{C}$ for $10 \mathrm{~min}$. The supernatant was diluted $1: 4$ with $0.25 \mathrm{M}$ sucrose solution. Three tubes were prepared for each experimental sample containing the following: $0.3 \mathrm{ml}$ of $0.1 \%$ histidine solution, $0.1 \mathrm{ml}$ $0.25 \mathrm{M}$ sucrose solution, and $0.1 \mathrm{ml}$ of diluted sample. At $15 \mathrm{~s}$ intervals each tube was placed into a $37^{\circ} \mathrm{C}$ water bath 
Zhao, Gao, Zhang, Li, Lv, Yu, Gao and Zhou

Table 2 The effects of in ovo feeding (IOF) of creatine pyruvate (CrPyr) on embryo characteristics on $19^{\text {th }}$ day of incubation (19 E), hatchability, hatching weight and BW at 3, 7 and 21 days post-hatch of broilers

\begin{tabular}{lccccccr}
\hline \hline & \multicolumn{7}{c}{ Treatments $^{1}$} \\
\cline { 2 - 5 } Items & Control & Saline & CrPyr $_{3}$ & CrPyr $_{6}$ & CrPyr $_{12}$ & SEM & $P$-value \\
\hline EW (g) & 56.9 & 56.6 & 58.0 & 58.8 & 58.4 & 0.5 & 0.499 \\
YBW (g) & $42.7^{\mathrm{b}}$ & $43.1^{\mathrm{b}}$ & $45.2^{\mathrm{a}}$ & $45.5^{\mathrm{a}}$ & $45.1^{\mathrm{a}}$ & 0.3 & 0.002 \\
YSW (g) & $9.3^{\mathrm{b}}$ & $9.5^{\mathrm{b}}$ & $10.6^{\mathrm{a}}$ & $10.9^{\mathrm{a}}$ & $10.7^{\mathrm{a}}$ & 0.2 & 0.001 \\
HF (\%) & 90.0 & 89.4 & 89.3 & 89.4 & $89.7^{\mathrm{a}}$ & 0.3 & 0.962 \\
HW (g) & $44.2^{\mathrm{b}}$ & $44.1^{\mathrm{b}}$ & $44.3^{\mathrm{b}}$ & $44.5^{\mathrm{b}}$ & $45.2^{\mathrm{a}}$ & 0.1 & 0.007 \\
BW at 3 days (g) & $60.9^{\mathrm{b}}$ & $60.7^{\mathrm{b}}$ & $60.3^{\mathrm{b}}$ & $61.0^{\mathrm{b}}$ & $63.4^{\mathrm{a}}$ & 0.4 & 0.047 \\
BW at 7 days (g) & $139.7^{\mathrm{b}}$ & $139.0^{\mathrm{b}}$ & $138.9^{\mathrm{b}}$ & $138.6^{\mathrm{b}}$ & $143.7^{\mathrm{a}}$ & 0.6 & 0.020 \\
BW at 21 days (g) & $775.5^{\mathrm{b}}$ & $780.8^{\mathrm{b}}$ & $794.0^{\mathrm{b}}$ & $816.9^{\mathrm{a}}$ & $832.6^{\mathrm{a}}$ & 4.8 & $<0.001$ \\
\hline \hline
\end{tabular}

$\mathrm{EW}=$ egg weight on $19 \mathrm{E}$; $\mathrm{YBW}=$ weight of embryos with the yolk sac on $19 \mathrm{E} ; \mathrm{YSW}=$ yolk sac weight on $19 \mathrm{E} ; \mathrm{HF}=$ hatchability of fertilized eggs (both male and female); $\mathrm{HW}=$ hatching weight of male chicks.

The results are presented by mean values and the SEM.

${ }_{\mathrm{a}, \mathrm{b}}$ Means within a row with different superscript letters are different at $P<0.05$.

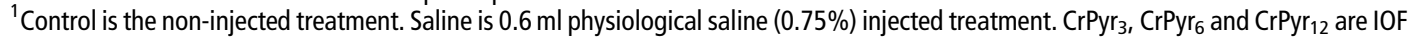
treatments injected with $0.6 \mathrm{ml}$ physiological saline (0.75\%) containing 3,6 or $12 \mathrm{mg}$ CrPyr per egg.

for a total incubation time of $10 \mathrm{~min}$. A volume of $0.1 \mathrm{ml}$ of $0.1 \mathrm{M}$ glucose-6-phosphate solution was added to two tubes of the triplicate set before incubation, and the remaining tube of the triplicate served as a sample blank. After $10 \mathrm{~min}$ of incubation, $1 \mathrm{ml}$ of $10 \% \mathrm{C}_{2} \mathrm{HCl}_{3} \mathrm{O}_{2}$ was added to each tube. For the sample blanks, $0.1 \mathrm{ml}$ of $0.1 \mathrm{M}$ glucose-6phosphate solution was added. Subsequently, the inorganic phosphate levels were measured using phosphate assay kits (Nanjing Jiancheng Bioengineering Institute, Nanjing, China) according to the instructions of the manufacturer. The activity of glucose-6-phosphatase was calculated and expressed in micromoles of substrate hydrolyzed per minute per milligram of protein in the tissues $(\mu \mathrm{mol} / \mathrm{min}$ per $\mathrm{mg}$ of protein).

\section{Statistical analysis}

Data analysis was performed by one-way ANOVA using SAS statistical software (version 8.02, SAS Institute Inc., Cary, NC, USA). In animal incubation and husbandry trials, the incubator trays and raising cages per treatment served as the experimental unit $(n=8)$. Therefore, the data on total and relative weight of pectoral muscle, activities of creatine kinase and glucose-6-phosphatase and the concentrations of glycogen, glucose, $\mathrm{Cr}$, $\mathrm{PCr}$ were analyzed using the individual embryo or broiler as the experimental unit $(n=8)$. Differences among treatments were examined using Duncan's multiple range tests. The means and pooled standard error of means were presented and differences were considered to be significant at $P<0.05$.

\section{Results}

Embryo characteristics, hatchability, hatching weight and body weight

Injection treatment had no significant effect on the egg weight at $19 \mathrm{E}(P>0.05$, Table 2$)$. However, compared with the control and saline groups, all IOF of CrPyr groups increased the weight of embryos with the yolk sac, as well as the yolk sac weight at $19 \mathrm{E}(P<0.05)$. No difference on hatchability was observed among treatments $(P>0.05)$. The hatching weight and BW of birds in $\mathrm{CrPyr}_{12}$ were significantly elevated compared with the control and saline groups at 3 and 7 days post-hatch $(P<0.05)$. On 21 days post-hatch, the BW was $5.34 \%$ and $7.37 \%$ greater in $\mathrm{CrPyr}_{6}$ and $\mathrm{CrPyr}_{12}$ than the control group $(P<0.05)$, respectively.

\section{Growth performance}

Birds showed similar growth performance among treatments during 1 to 7 days post-hatch $(P>0.05$, Table 3$)$. However, the chickens in $\mathrm{CrPyr}_{6}$ and $\mathrm{CrPyr}_{12}$ exhibited higher BW gain and feed intake than the control and saline groups during 8 to 21 days post-hatch and the entire experiment period $(P<0.05)$. All treatments had similar feed/gain ratio irrespective of the growth period $(P>0.05)$.

Pectoral muscle weight and relative pectoral muscle weight As shown in Table 4, significant increases in total and relative weight of pectoral muscle were observed in $\mathrm{CrPyr}_{6}$ and $\mathrm{CrPyr}_{12}$ treatments compared with the control and saline groups at $19 \mathrm{E}$, hatch, 3 and 21 days of age $(P<0.05)$. In addition, the birds in $\mathrm{CrPyr}_{12}$ gained greater total and relative weight of pectoral muscle than the control and saline groups at 7 days post-hatch $(P<0.05)$.

\section{Concentrations of glycogen and glucose in liver and pectoral muscle}

The concentrations of glycogen were increased in $\mathrm{CrPyr}_{6}$ and $\mathrm{CrPyr}_{12}$ in comparison with the control, saline and $\mathrm{CrPyr}_{3}$ groups in liver at $19 \mathrm{E}(P<0.05$, Figure $1 \mathrm{~A})$. All groups showed a pattern of reduction in liver glycogen reserves as the embryo approached hatch, whereas the glycogen concentrations of hatchlings in $\mathrm{CrPyr}_{6}$ and $\mathrm{CrPyr}_{12}$ were 1.79-fold and 
In ovo feeding of creatine pyruvate in embryos

Table 3 The effects of in ovo feeding (IOF) of creatine pyruvate (CrPyr) on the growth performance of broilers

\begin{tabular}{|c|c|c|c|c|c|c|c|}
\hline \multirow[b]{2}{*}{ Items } & \multicolumn{5}{|c|}{ Treatments $^{1}$} & \multirow[b]{2}{*}{ SEM } & \multirow[b]{2}{*}{$P$-value } \\
\hline & Control & Saline & $\mathrm{CrPyr}_{3}$ & $\mathrm{CrPyr}_{6}$ & $\mathrm{CrPyr}_{12}$ & & \\
\hline \multicolumn{8}{|l|}{ BWG (g/bird) } \\
\hline 1 to 7 days & 97.0 & 96.1 & 96.1 & 95.9 & 98.8 & 0.6 & 0.441 \\
\hline 8 to 21 days & $635.8^{b}$ & $641.8^{b}$ & $655.1^{\mathrm{b}}$ & $678.3^{a}$ & $689.0^{a}$ & 4.7 & $<0.001$ \\
\hline 1 to 21 days & $732.8^{c}$ & $738.0^{c}$ & $751.1^{b c}$ & $774.2^{\mathrm{ab}}$ & $787.8^{\mathrm{a}}$ & 4.8 & $<0.001$ \\
\hline \multicolumn{8}{|l|}{ Fl (g/bird) } \\
\hline 1 to 7 days & 108.8 & 107.4 & 109.0 & 108.3 & 110.9 & 0.7 & 0.643 \\
\hline 8 to 21 days & $925.1^{\mathrm{b}}$ & $928.9^{b}$ & $925.9^{b}$ & $963.7^{\mathrm{a}}$ & $971.6^{\mathrm{a}}$ & 5.8 & 0.009 \\
\hline 1 to 21 days & $1033.8^{b}$ & $1036.3^{b}$ & $1034.9^{b}$ & $1072.1^{\mathrm{a}}$ & $1082.5^{\mathrm{a}}$ & 6.0 & 0.008 \\
\hline \multicolumn{8}{|l|}{$F: G(g: g)$} \\
\hline 1 to 7 days & 1.12 & 1.12 & 1.14 & 1.13 & 1.12 & 0.01 & 0.956 \\
\hline 8 to 21 days & 1.46 & 1.45 & 1.41 & 1.42 & 1.41 & 0.01 & 0.417 \\
\hline 1 to 21 days & 1.41 & 1.40 & 1.38 & 1.39 & 1.37 & 0.01 & 0.506 \\
\hline
\end{tabular}

BWG = BW gain; $\mathrm{FI}=$ feed intake; $\mathrm{F}: \mathrm{G}=$ feed : gain

The results are presented by mean values and the SEM.

$a, b, c$ Means within a row with different superscript letters are different at $P<0.05$.

${ }^{1}$ Control is the non-injected treatment. Saline is $0.6 \mathrm{ml}$ physiological saline $(0.75 \%)$ injected treatment. $\mathrm{CrPyr}_{3} \mathrm{CrPyr}_{6}$ and $\mathrm{CrPyr}_{12}$ are IOF treatments injected with $0.6 \mathrm{ml}$ physiological saline (0.75\%) containing 3, 6 or $12 \mathrm{mg}$ CrPyr per egg.

Table 4 The effects of in ovo feeding (IOF) of creatine pyruvate (CrPyr) on the pectoral muscle weight and relative pectoral muscle weight of embryos and broilers on $19^{\text {th }}$ day of incubation $(19 \mathrm{E})$, the day of hatch, and 3, 7 and 21 days post-hatch

\begin{tabular}{|c|c|c|c|c|c|c|c|}
\hline \multirow[b]{2}{*}{ Items } & \multicolumn{5}{|c|}{ Treatments $^{1}$} & \multirow[b]{2}{*}{ SEM } & \multirow[b]{2}{*}{$P$-value } \\
\hline & Control & Saline & $\mathrm{CrPyr}_{3}$ & $\mathrm{CrPyr}_{6}$ & $\mathrm{CrPyr}_{12}$ & & \\
\hline \multicolumn{8}{|c|}{ Pectoral muscle weight (g) } \\
\hline $19 \mathrm{E}$ & $0.74^{\mathrm{b}}$ & $0.76^{\mathrm{b}}$ & $0.78^{b}$ & $0.89^{\mathrm{a}}$ & $0.92^{\mathrm{a}}$ & 0.02 & $<0.001$ \\
\hline Hatch & $0.60^{\mathrm{b}}$ & $0.63^{b}$ & $0.65^{b}$ & $0.84^{\mathrm{a}}$ & $0.88^{\mathrm{a}}$ & 0.02 & $<0.001$ \\
\hline 3 days & $1.05^{c}$ & $1.10^{c}$ & $1.15^{\mathrm{bc}}$ & $1.24^{\mathrm{ab}}$ & $1.31^{\mathrm{a}}$ & 0.02 & $<0.001$ \\
\hline 7 days & $7.21^{\mathrm{b}}$ & $7.07^{b}$ & $7.28^{\mathrm{b}}$ & $7.30^{\mathrm{b}}$ & $8.02^{\mathrm{a}}$ & 0.10 & 0.027 \\
\hline 21 days & $113.60^{c}$ & $112.69^{c}$ & $117.19^{c}$ & $133.49^{b}$ & $141.42^{\mathrm{a}}$ & 2.08 & $<0.001$ \\
\hline \multicolumn{8}{|c|}{ Relative pectoral muscle weight (\%) } \\
\hline $19 \mathrm{E}$ & $2.20^{\mathrm{b}}$ & $2.23^{b}$ & $2.27^{b}$ & $2.59^{\mathrm{a}}$ & $2.70^{\mathrm{a}}$ & 0.05 & $<0.001$ \\
\hline Hatch & $1.40^{\mathrm{b}}$ & $1.45^{\mathrm{b}}$ & $1.49^{b}$ & $1.88^{\mathrm{a}}$ & $1.96^{\mathrm{a}}$ & 0.04 & $<0.001$ \\
\hline 3 days & $1.92^{\mathrm{b}}$ & $1.96^{\mathrm{b}}$ & $2.07^{\mathrm{ab}}$ & $2.19^{a}$ & $2.26^{\mathrm{a}}$ & 0.03 & 0.002 \\
\hline 7 days & $5.91^{\mathrm{b}}$ & $5.92^{b}$ & $6.18^{\mathrm{ab}}$ & $6.19^{a b}$ & $6.25^{a}$ & 0.05 & 0.049 \\
\hline 21 days & $15.42^{c}$ & $15.25^{c}$ & $15.21^{c}$ & $16.16^{b}$ & $16.77^{a}$ & 0.13 & $<0.001$ \\
\hline
\end{tabular}

Relative pectoral muscle weight $=$ pectoral muscle weight as a percentage of BW.

The results are presented by mean values and the SEM.

$a, b, c$ Means within a row with different superscript letters are different at $P<0.05$.

${ }^{1}$ Control is the non-injected treatment. Saline is $0.6 \mathrm{ml}$ physiological saline $(0.75 \%)$ injected treatment. $\mathrm{CrPyr}_{3} \mathrm{CrPyr}_{6}$ and $\mathrm{CrPyr}_{12}$ are IOF treatments injected with $0.6 \mathrm{ml}$ physiological saline (0.75\%) containing 3, 6 or $12 \mathrm{mg} \mathrm{CrPyr}$ per egg.

2.13-fold of the control at hatch $(P<0.05)$. Meanwhile, these two groups had higher concentration of glucose than other samples at $19 \mathrm{E}$ and hatch in liver $(P<0.05$, Figure $1 \mathrm{C})$. At 3 days post-hatch, birds in $\mathrm{CrPyr}_{6}$ and $\mathrm{CrPyr}_{12}$ had higher concentration of glycogen in liver than other groups, whereas this result maintained at 7 days post-hatch in $\mathrm{CrPyr}_{12}$ $(P<0.05)$. Neither glycogen nor glucose concentration in pectoral muscle was altered among treatments at any of the time points measured $(P>0.05$, Figure $1 \mathrm{~B}$ and $\mathrm{D})$.

\section{Concentrations of creatine and phosphocreatine in pectoral muscle}

There were significant positive effects on concentration of $\mathrm{Cr}$ in pectoral muscle compared with the control and saline groups in all IOF of CrPyr groups at $19 \mathrm{E}$ and hatch $(P<0.05)$, and the results were also found in $\mathrm{CrPyr}_{12}$ group on 3 days post-hatch $(P<0.05$, Figure $2 A)$. In addition, the concentrations of $\mathrm{PCr}$ were higher in all CrPyr-treated groups than the control and saline groups at $19 \mathrm{E}(P<0.05$, Figure $2 \mathrm{~B})$. 

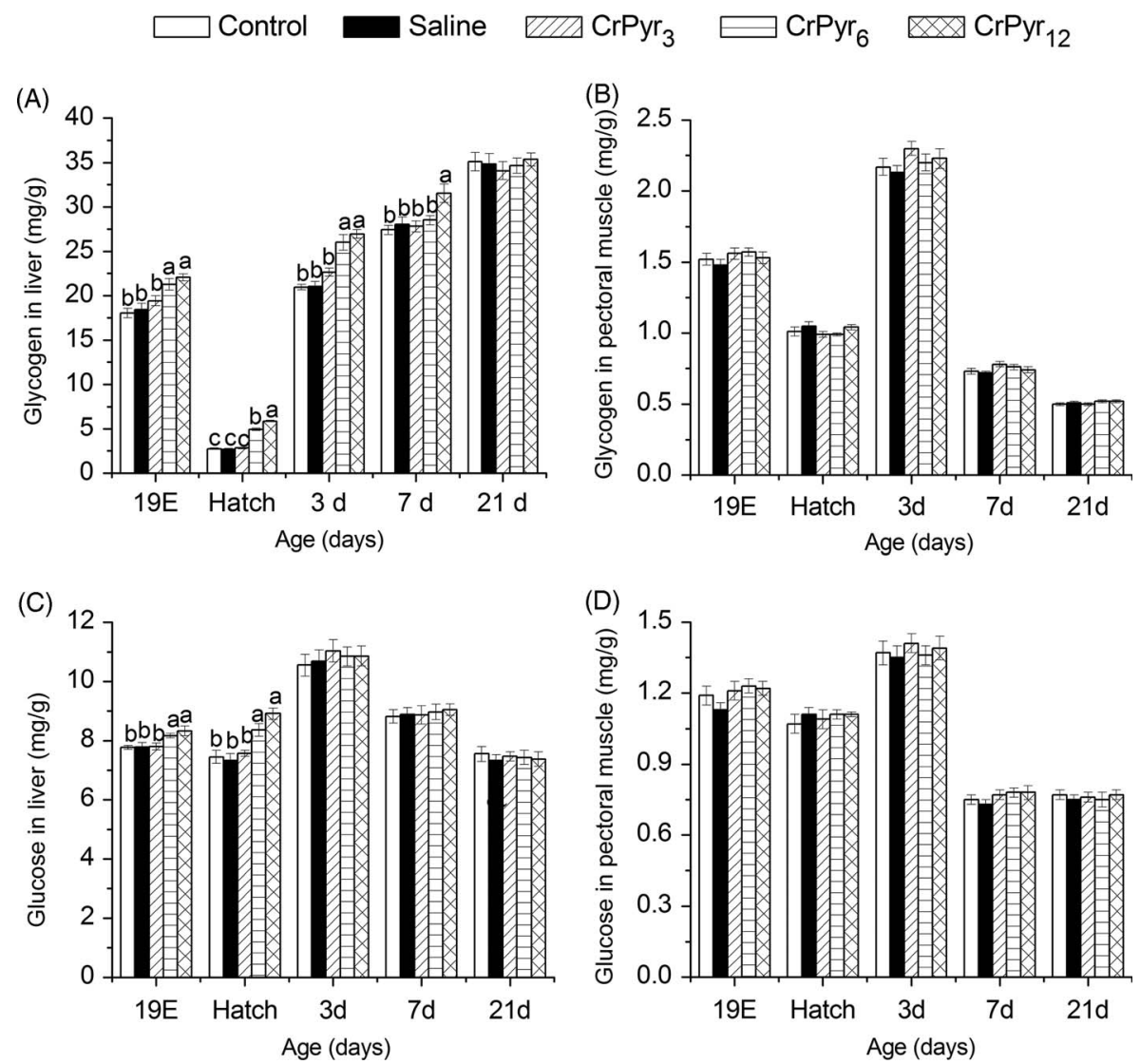

Figure 1 The effects of in ovo feeding (IOF) of creatine pyruvate (CrPyr) on the concentrations of glycogen and glucose in liver ((A) and (C)) and pectoral muscle $((B)$ and $(D))$ of embryos and broilers on $19^{\text {th }}$ day of incubation (19E), the day of hatch, 3, 7 and 21 days post-hatch. Control is the non-injected treatment. Saline is $0.6 \mathrm{ml}$ physiological saline (0.75\%) injected treatment. $\mathrm{CrPyr}_{3}, \mathrm{CrPyr}_{6}$ and $\mathrm{CrPyr}_{12}$ are IOF treatments injected with $0.6 \mathrm{ml}$ physiological saline $(0.75 \%)$ containing 3,6 or $12 \mathrm{mg}$ CrPyr per egg. All data are represented as the mean value \pm SE of eight sample embryos or birds per treatment. $a, b, c$ Different letters within the same time points indicate significant differences between the five treatments $(P<0.05)$.

\begin{abstract}
Activities of creatine kinase and glucose-6-phosphatase A notable increase in activity of creatine kinase in pectoral muscle was observed in all IOF of CrPyr groups at 19 $\mathrm{E}$ when compared with the control and saline groups $(P<0.05$, Table 5). Simultaneously, the activities of glucose6-phosphatase in liver in $\mathrm{CrPyr}_{6}$ and $\mathrm{CrPyr}_{12}$ groups were higher than other treatments $(P<0.05)$.
\end{abstract}

\section{Discussion}

The hatchability of poultry, as one of the major determinants of profitability in a hatchery enterprise, is influenced by many factors such as genetics, breeder hen age, egg size and incubation conditions (Kadam et al., 2013). As reported in previous study, IOF of $0.5 \mathrm{ml} / \mathrm{egg}$ of carbohydrate (maltose, sucrose and dextrin mixture in a proportion of $1: 1: 8$ ) varied from 50 to $250 \mathrm{mg} / \mathrm{egg}$ had no significant effect on hatchability of broilers (Shafey et al., 2012). On the contrary, Dong et al. (2013) asserted that injection of $0.2 \mathrm{ml} / \mathrm{egg}$ of $4.5 \%$ maltose $+4.5 \%$ sucrose into amnion of pigeon eggs reduced hatchability and concluded that the concentration of injection solution should be limited in order to prevent excessive energy metabolism of the embryos. Another research claimed that hatchability was negatively related to injection volume of carbohydrate solution (Zhai et al., 2011b). These results suggested that the effects of IOF on hatchability might be attributed to other profound factors including the solution formulation, concentration and appropriate injection volume. No significant difference in hatchability was observed among treatments, suggesting that the injection dose in the present study was safe.

This study indicated that IOF of $12 \mathrm{mg} / \mathrm{egg}$ CrPyr improved chick hatching weight and this advantage was sustained up to 21 days of age at least, which agreed the results observed in turkeys, ducks and domestic pigeons (Foye et al., 2006b; Chen et al., 2009; Dong et al., 2013). In fact, hatching weight is a vital indicator of marketing weight in poultry, whereas this correlation may differ among strains (Sklan et al., 2003; Willemsen et al., 2008). Wen et al. (2014) reported that $6.6 \mathrm{~g}$ of increase in hatching weight of Arbor Acres broiler chickens led to $252 \mathrm{~g}$ of increase in BW at 42 days post-hatch. However, our research showed that a $0.98 \mathrm{~g}$ difference in $\mathrm{BW}$ at hatch due to IOF of $12 \mathrm{mg} / \mathrm{egg}$ CrPyr resulted in $57.12 \mathrm{~g}$ of increase in BW at 21 days post-hatch. In addition, Kornasio et al. (2011) reported that IOF of carbohydrates and $\beta$-hydroxy- $\beta$-methylbutyrate increased the pectoral muscle weight of broilers on 35 days post-hatch. The broilers in 6 

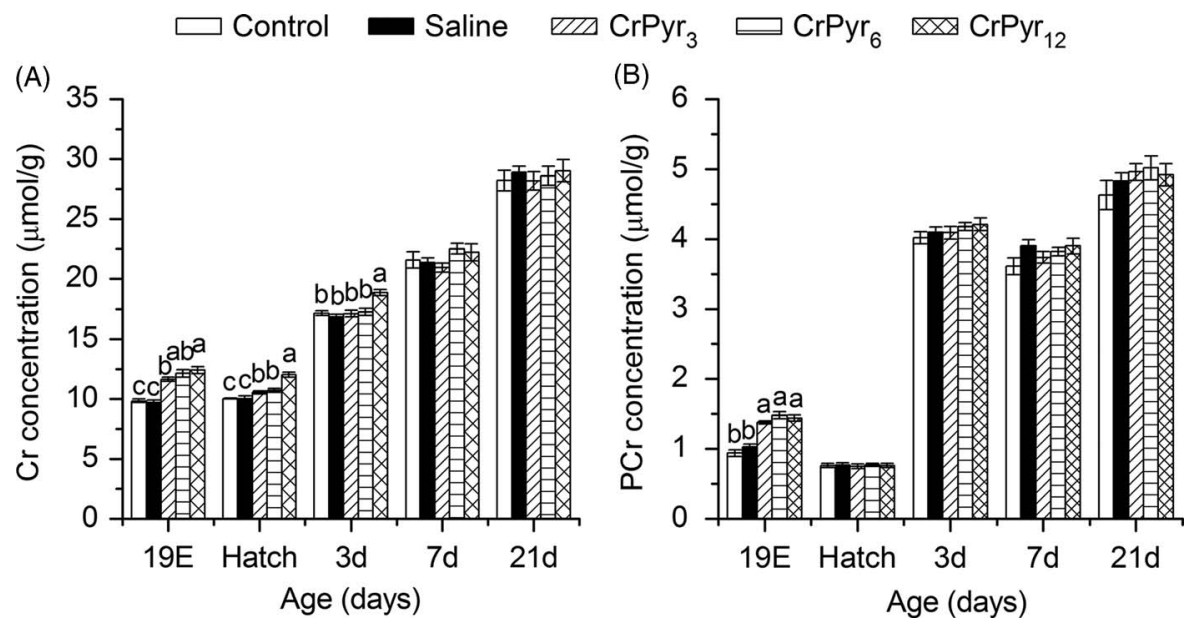

Figure 2 The effects of in ovo feeding (IOF) of creatine pyruvate (CrPyr) on the concentrations of creatine ( $\mathrm{Cr}$ ) and phosphocreatine (PCr) in pectoral muscle $((A)$ and $(B))$ of embryos and broilers on $19^{\text {th }}$ day of incubation (19E), the day of hatch, 3, 7 and 21 days post-hatch. Control is the non-injected treatment. Saline is $0.6 \mathrm{ml}$ physiological saline (0.75\%) injected treatment. CrPyr ${ }_{3}, \mathrm{CrPyr}_{6}$ and $\mathrm{CrPyr}_{12}$ are IOF treatments injected with $0.6 \mathrm{ml}$ physiological saline $(0.75 \%)$ containing 3,6 or $12 \mathrm{mg}$ CrPyr per egg. All data are represented as the mean value \pm SE of eight sample embryos or birds per treatment. $a, b, c$ Different letters within the same time points indicate significant differences between the five treatments $(P<0.05)$.

Table 5 The effects of in ovo feeding (IOF) of creatine pyruvate (CrPyr) on the activities of creatine kinase in pectoral muscle and glucose-6-phosphatase in liver of embryos and broilers on $19^{\text {th }}$ day of incubation (19E), the day of hatch, and 3, 7 and 21 days post-hatch

\begin{tabular}{|c|c|c|c|c|c|c|c|}
\hline \multirow[b]{2}{*}{ Items } & \multicolumn{5}{|c|}{ Treatments $^{1}$} & \multirow[b]{2}{*}{ SEM } & \multirow[b]{2}{*}{$P$-value } \\
\hline & Control & Saline & $\mathrm{CrPyr}_{3}$ & $\mathrm{CrPyr}_{6}$ & $\mathrm{CrPyr}_{12}$ & & \\
\hline \multicolumn{8}{|c|}{ Activity of creatine kinase (U/mg of protein) } \\
\hline $19 \mathrm{E}$ & $4.41^{\mathrm{c}}$ & $4.50^{c}$ & $4.98^{b}$ & $5.29^{\mathrm{ab}}$ & $5.57^{\mathrm{a}}$ & 0.09 & $<0.001$ \\
\hline Hatch & 5.66 & 5.85 & 5.57 & 5.94 & 6.03 & 0.07 & 0.252 \\
\hline 3 days & 6.58 & 6.45 & 6.56 & 6.91 & 6.76 & 0.07 & 0.308 \\
\hline 7 days & 9.88 & 9.85 & 9.99 & 9.88 & 9.92 & 0.11 & 0.996 \\
\hline 21 days & 3.97 & 3.89 & 4.07 & 4.03 & 3.98 & 0.05 & 0.785 \\
\hline \multicolumn{8}{|c|}{ Activity of glucose- 6 -phosphatase ( $\mu \mathrm{mol} / \mathrm{min}$ per $\mathrm{mg}$ of protein) } \\
\hline $19 \mathrm{E}$ & $0.121^{\mathrm{b}}$ & $0.125^{b}$ & $0.129^{b}$ & $0.152^{a}$ & $0.161^{\mathrm{a}}$ & 0.003 & $<0.001$ \\
\hline Hatch & 0.114 & 0.110 & 0.121 & 0.119 & 0.120 & 0.002 & 0.235 \\
\hline 3 days & 0.068 & 0.065 & 0.067 & 0.070 & 0.068 & 0.001 & 0.584 \\
\hline 7 days & 0.061 & 0.059 & 0.062 & 0.061 & 0.063 & 0.001 & 0.724 \\
\hline 21 days & 0.068 & 0.066 & 0.070 & 0.069 & 0.072 & 0.001 & 0.460 \\
\hline
\end{tabular}

The results are presented by mean values and the SEM.

a,b,c Means within a row with different superscript letters are different at $P<0.05$.

${ }^{1}$ Control is the non-injected treatment. Saline is $0.6 \mathrm{ml}$ physiological saline $(0.75 \%)$ injected treatment. CrPyr3, $\mathrm{CrPyr} 6$ and $\mathrm{CrPyr} 12$ are IOF treatments injected with $0.6 \mathrm{ml}$ physiological saline $(0.75 \%)$ containing 3,6 or $12 \mathrm{mg}$ CrPyr per egg.

and $12 \mathrm{mg} /$ egg CrPyr group showed a significant increase in the pectoral muscle weight and relative pectoral muscle weight on 21 days post-hatch in the present study. Based on the current results, if the benefits persist to slaughter age, it is suggested that IOF of appropriate nutrients might be an effective technique to stimulate avian embryo development, increase the market weight and pectoral muscle weight of growing chickens.

The glucose, mainly stored as glycogen in the liver and glycolytic muscles of embryos, is preferentially utilized as energy source over lipid and protein because of the limitation of oxygen availability especially during late incubation
(Moran, 2007). Nevertheless, it is well known that the demand for glucose is high and the primary mechanism of glucose production depends on hepatic gluconeogenesis using the substrates of lactate (Kobayashi et al., 1989) and glucogenic amino acid from the amnion and muscles in the avian embryos and neonates (Edwards et al., 1997). In the current study, the data in non-injected control group proved that the depletion of energy reserves might occur in late-term chick embryos, as illustrated by exhausting up to $15 \%$ of liver glycogen and $66 \%$ of pectoral glycogen concentrations from $19 \mathrm{E}$ to hatch. In addition, our results indicated that IOF of 6 and $12 \mathrm{mg} / \mathrm{egg}$ CrPyr raised liver glucose and glycogen 
accumulation at $19 \mathrm{E}$ and hatch, which were consistent with the findings of Foye et al. (2006b) and Chen et al. (2010). These results implied that IOF of CrPyr might have a substrate-mediated effect on the concentrations of glucose and glycogen in liver, due to $\mathrm{CrPyr}$ providing pyruvate as the possible substrates for hepatic gluconeogenesis. As expected, the activity of liver glucose-6-phosphatase enzyme, one of the key enzymes in the gluconeogenesis pathway, was also increased in embryos IOF of CrPyr solutions at $19 \mathrm{E}$. Another similar study reported that there was a high positive correlation between BW and the concentration of glycogen in liver (Tangara et al., 2010), suggesting that the improvement of the BW of broilers in this study could be partially attributed to the higher concentration of glycogen in liver.

In addition, the $\mathrm{Cr}-\mathrm{PCr}$ system has been reported to maintain energy homeostasis by buffering ADP and ATP ratios via a freely reversible reaction catalyzed by creatine kinase in muscles (Allen, 2012). The creatine monohydrate supplementation in diet has been recently reported to elevate the concentration of $\mathrm{Cr}$, activity of creatine kinase (Li et al., 2016) and the level of PCr (Young et al., 2007) in muscle of pigs. Wang et al. (2015) also showed that dietary $1200 \mathrm{mg} / \mathrm{kg}$ creatine monohydrate supplementation increased the concentrations of $\mathrm{Cr}$ and $\mathrm{PCr}$ in pectoral muscle of $3 \mathrm{~h}$ transported broilers in comparison to a 45 min transported control. Similarly, the present study proved that IOF of CrPyr increased the concentrations of $\mathrm{Cr}$ and $\mathrm{PCr}$ at $19 \mathrm{E}$, which could provide more ATP to avoid energy imbalance when energy demand increased at hatch. Therefore, this additional energy sources resulting from $\mathrm{Cr}-\mathrm{PCr}$ system probably, at least in part, could improve the energy status of embryos and hatchlings, which was useful for development of BW. Uni et al. (2005) suggested that IOF of carbohydrates and $\beta$-hydroxy- $\beta$-methylbutyrate in late-term embryos could improve liver glycogen by two to five fold and elevate relative breast muscle size by $6 \%$ to $8 \%$ on the day of hatch. In the present study, compared with non-injected birds, the total and relative weight of pectoral muscle of broilers in 6 and $12 \mathrm{mg} / \mathrm{egg}$ CrPyr-injected groups were increased by $19.89 \mathrm{~g}$ $(4.80 \%)$ and $27.82 \mathrm{~g}(8.75 \%)$ at 21 days of age, respectively. It is reasonable to assume that the higher energy reserves including glycogen and $\mathrm{PCr}$ probably reduce the need for glucose synthesis via gluconeogenesis from muscle proteins, resulting in higher pectoral muscle weight of embryos and broilers. Moreover, the yolk sac weight was increased in CrPyr groups in comparison with the control and saline groups at $19 \mathrm{E}$, which is consistent with the results of Zhai et al. (2011b) and Zhang et al. (2016). These findings suggested that the embryos in late embryonic stage could utilize the exogenous energy nutrients thereby sparing the yolk sac nutrient utilization. The higher residual yolks may be beneficial to the maintenance and growth of hatched broilers (Zhai et al., 2011a).

In contrast to glycogen dynamics in liver, there was no significant change in glycogen reserves of pectoral muscle in this study. Similarly, Foye et al. (2006a) and Tangara et al.
(2010) maintained that IOF of protein or arginine alone also had no influence on muscle glycogen levels. Conversely, another study claimed that IOF of carbohydrates (simpler sugars) into amnion of pigeon eggs increased the glycogen reserves in pectoral muscle (Dong et al., 2013). This discrepancy may be explained by the lack of glucose-6phosphatase enzyme needed for gluconeogenesis and the requirement of insulin for uptake of glucose from the blood in skeletal muscle (Foye et al., 2006a). It has been noted that IOF of carbohydrates could accelerate the uptake and storage of glucose in the form of glycogen in the muscles mainly through the release of insulin (Foye et al., 2006b). Hence, IOF of a part of non-carbohydrate nutrients, such as protein and arginine, may not stimulate the secretion of insulin, thus, the glucose produced from hepatic gluconeogenesis is mainly stored as glycogen in liver rather than pectoral muscle (Foye et al., 2006a), which corroborate with the effects of CrPyr in this study. In addition, the glycogen concentration in liver showed an increasing trend during 3 to 21 days post-hatch, whereas the glycogen concentration in muscle decreased from 3 to 7 days and kept nearly constant to 21 days posthatch. The reason may be attributed to the different physiological functions of glycogen in liver and muscle. The liver glycogen mainly regulates the stability of blood glucose to provide energy for multiple organs. However, the muscle glycogen is primary used to generate ATP for muscle protein synthesis (very high in the breast muscle of 1- to 3-week-old chickens) and muscular contractions under the oxidative or glycolytic pathways.

In conclusion, the present study demonstrated that IOF of CrPyr on 17.5 days of incubation was beneficial to increase the concentrations of glucose and glycogen in liver, as well as the concentrations of $\mathrm{Cr}$ and $\mathrm{PCr}$ in pectoral muscle, which may contribute to the improvement of energy status of embryos and hatchlings, and subsequently improved the BW and pectoral muscle weight until the end of the experiments at 21 days post-hatch in broilers. The appropriate injection level of CrPyr was recommended at $12 \mathrm{mg} / \mathrm{egg}$ in the present study.

\section{Acknowledgments}

This research was supported by the National Natural Science Foundation of China (no. 31572425) and the National Key Research and Development Program of China (no. 2016YFD0500501).

\section{References}

Allen PJ 2012. Creatine metabolism and psychiatric disorders: does creatine supplementation have therapeutic value? Neuroscience and Biobehavioral Reviews 36, 1442-1462.

Burke WH 1994. Sex differences in weight of turkey embryos. Poultry Science 73, 749-753.

Chen J, Huang JZ, Deng J, Ma HT and Zou SX 2012. Use of comparative proteomics to identify the effects of creatine pyruvate on lipid and protein metabolism in broiler chickens. The Veterinary Journal 193, 514-521. 
Chen J, Wang M, Kong Y, Ma H and Zou S 2011. Comparison of the novel compounds creatine and pyruvate on lipid and protein metabolism in broiler chickens. Animal 5, 1082-1089.

Chen W, Wang R, Wan HF, Xiong XL, Peng P and Peng J 2009. Influence of in ovo injection of glutamine and carbohydrates on digestive organs and pectoralis muscle mass in the duck. British Poultry Science 50, 436-442.

Chen W, Xu J, Tangara M and Peng J 2010. Effects of in ovo injecting disaccharides and alanyl-glutamine dipeptide on the energy status in duck embryos and neonates. Animal Reproduction Science 122, 29-35.

Donaldson WE and Christensen VL 1991. Dietary carbohydrate level and glucose metabolism in turkey poults. Comparative Biochemistry and Physiology Part A: Physiology 98, 347-350.

Dong XY, Jiang YJ, Wang MQ, Wang YM and Zou XT 2013. Effects of in ovo feeding of carbohydrates on hatchability, body weight, and energy status in domestic pigeons (Columba livia). Poultry Science 92, 2118-2123.

Edwards HM III, Baker DH, Fernandez SR and Parsons CM 1997. Maintenance threonine requirement and efficiency of its use for accretion of whole-body threonine and protein in young chicks. British Journal of Nutrition 78, 111-119. Foye OT, Uni Z and Ferket PR 2006a. Effects of in ovo feeding egg white protein, $\beta$-hydroxy- $\beta$-methylbutyrate, and carbohydrates on glycogen status and neonatal growth of turkeys. Poultry Science 851, 185-1192.

Foye OT, Uni Z, McMurtry JP and Ferket PR 2006b. The effects of amniotic nutrient administration, 'in ovo feeding' of arginine and/or $\beta$-hydroxy- $\beta$ methylbutyrate (HMB) on insulin-like growth factors, energy metabolism and growth in turkey poults. International Journal of Poultry Science 5 , 309-317.

Kadam MM, Barekatain MR, Bhanja SK and lji PA 2013. Prospects of in ovo feeding and nutrient supplementation for poultry: the science and commercial applications-a review. Journal of the Science of Food and Agriculture 93, 3654-3661.

Kobayashi T, Iwai H, Uchimoto R, Ohta M, Shiota M and Sugano T 1989. Gluconeogenesis in perfused livers from dexamethasone-treated chickens. American Journal of Physiology-Regulatory, Integrative and Comparative Physiology 256, R907-R914.

Kornasio R, Halevy O, Kedar 0 and Uni Z 2011. Effect of in ovo feeding and its interaction with timing of first feed on glycogen reserves, muscle growth, and body weight. Poultry Science 90, 1467-1477.

Lamot DM, van de Linde IB, Molenaar R, van der Pol CW, Wijtten PJA, Kemp B and van den Brand $\mathrm{H}$ 2014. Effects of moment of hatch and feed access on chicken development. Poultry Science 93, 2604-2614.

Li JL, Guo ZY, Li YJ, Zhang L, Gao F and Zhou GH 2016. Effect of creatine monohydrate supplementation on carcass traits, meat quality and postmortem energy metabolism of finishing pigs. Animal Production Science 56, 48-54.

Moran ET Jr 2007. Nutrition of the developing embryo and hatchling. Poultry Science 86, 1043-1049.

Noy $Y$ and Uni Z 2010. Early nutritional strategies. World's Poultry Science Journal 66, 639-646.

Shafey TM, Alodan MA, Al-Ruqaie IM and Abouheif MA 2012. In ovo feeding of carbohydrates and incubated at a high incubation temperature on hatchability and glycogen status of chicks. South African Journal of Animal Science 42 210-220.
Sklan D, Heifetz S and Halevy 0 2003. Heavier chicks at hatch improves marketing body weight by enhancing skeletal muscle growth. Poultry Science 82 , 1778-1786.

Tangara M, Chen W, Xu J, Huang FR and Peng J 2010. Effects of in ovo feeding of carbohydrates and arginine on hatchability, body weight, energy metabolism and perinatal growth in duck embryos and neonates. British Poultry Science 51, 602-608.

Uni Z and Ferket RP 2004. Methods for early nutrition and their potential. World's Poultry Science Journal 60, 101-111.

Uni Z, Ferket PR, Tako E and Kedar 0 2005. In ovo feeding improves energy status of late-term chicken embryos. Poultry Science 84, 764-770.

Wang XF, Zhu XD, Li YJ, Liu Y, Li JL, Gao F, Zhou GH and Zhang L 2015. Effect of dietary creatine monohydrate supplementation on muscle lipid peroxidation and antioxidant capacity of transported broilers in summer. Poultry Science 94, 2797-2804.

Wen C, Wu P, Chen YP, Wang T and Zhou YM 2014. Methionine improves the performance and breast muscle growth of broilers with lower hatching weight by altering the expression of genes associated with the insulin-like growth factor-I signalling pathway. British Journal of Nutrition 111, 201-206.

Willemsen $H$, Debonne $M$, Swennen Q, Everaert $N$, Careghi C, Han $H_{t}$ Bruggeman V, Tona $\mathrm{K}$ and Decuypere E 2010. Delay in feed access and spread of hatch: importance of early nutrition. World's Poultry Science Journal 66, 177-188.

Willemsen $H$, Everaert $N$, Witters $A$, De Smit L, Debonne $M$, Verschuere $F$, Garain P, Berckmans D, Decuypere E and Bruggeman V 2008. Critical assessment of chick quality measurements as an indicator of posthatch performance. Poultry Science 87, 2358-2366.

Young JF, Bertram HC, Theil PK, Petersen AG, Poulsen KA, Rasmussen M Malmendal A, Nielsen NC, Vestergaard M and Oksbjerg N 2007. In vitro and in vivo studies of creatine monohydrate supplementation to Duroc and Landrace pigs. Meat Science 76, 342-351.

Zhai W, Gerard PD, Pulikanti R and Peebles ED 2011a. Effects of in ovo injection of carbohydrates on embryonic metabolism, hatchability, and subsequent somatic characteristics of broiler hatchlings. Poultry Science 90, 2134-2143.

Zhai W, Rowe DE and Peebles ED 2011b. Effects of commercial in ovo injection of carbohydrates on broiler embryogenesis. Poultry Science 90, 1295-1301.

Zhang L, Yue HY, Wu SG, Xu L, Zhang HJ, Yan HJ, Cao YL, Gong YS and Qi GH 2010. Transport stress in broilers. II. Superoxide production, adenosine phosphate concentrations, and mRNA levels of avian uncoupling protein, avian adenine nucleotide translocator, and avian peroxisome proliferatoractivated receptor- $\gamma$ coactivator- $1 \alpha$ in skeletal muscles. Poultry Science 89, 393-400.

Zhang L, Zhang HJ, Wang J, Wu SG, Qiao X, Yue HY, Yao JH and Qi GH 2014. Stimulation with monochromatic green light during incubation alters satellite cell mitotic activity and gene expression in relation to embryonic and posthatch muscle growth of broiler chickens. Animal 8, 86-93.

Zhang L, Zhu XD, Wang XF, Li JL, Gao F and Zhou GH 2016. Individual and combined effects of in-ovo injection of creatine monohydrate and glucose on somatic characteristics, energy status, and posthatch performance of broiler embryos and hatchlings. Poultry Science 95, 2352-2359. 